\title{
Cosmic Gamma Ray Bursts
}

\author{
A. JAniUK*, B. James And K. SAPOUntzis \\ Center for Theoretical Physics, Polish Academy of Sciences, \\ al. Lotników 32/46, 02-668 Warsaw, Poland \\ Doi: $10.12693 /$ APhysPolA.139.273 \\ *e-mail: agnes@cft.edu.pl
}

\begin{abstract}
Gamma ray bursts are astronomical phenomena detected at highest energies. The gamma ray photons carry energies on the order of mega-electronovolts and arrive to us from the point-like sources that are uniformly distributed in the sky. A typical burst has a form of a pulse that lasts for about a minute. As the Earth atmosphere is not transparent to the very high energy radiation, the bursts are detected by means of telescopes on-board satellites that are placed on the orbit. The total energetics of gamma ray burst events, which is given by the integrated energy flux by the detector unit area, implies that we are witnessing very powerful explosions, where an enormously great power is released within a short time. There is only one way to obtain such huge energies in cosmos: a disruption of a star.
\end{abstract}

topics: accretion, accretion disks, black hole physics, magnetohydrodynamics (MHD)

\section{Introduction}

Multi-wavelength and multi-messenger observations show that black holes are the central engines responsible for the most violent astrophysical events such as, for instance, active nuclei of galaxies, X-ray binaries, core-collapse supernovae or gamma-ray bursts. This central engine is subject to strong gravity, strong electromagnetic fields, and rotation. The governing physical laws of such engines are well-known (general relativistic magnetohydrodynamics, GRMHD) but are nonlinear, timedependent, and multidimensional. Thus, it is necessary to develop a numerical approach to simulate their evolution and observational appearance where a first-principles theory cannot be achieved.

In order to produce a gamma ray burst, the black hole is launching a jet of plasma. In these jets, plasma is expanding with ultra-relativistic velocities. Particles are accelerated in a strong magnetic field and produce high energy radiation. The jet is powered by the Blandford-Znajek mechanism which can extract energy from a rotating black hole [1]. This process requires a magnetized accretion disk with a strong poloidal magnetic field generated around a spinning black hole. The gammaray emission produced in the jet at large distances is not uniform and short time-scale variability suggests that it originates close to the black hole.

\section{Two classes of gamma ray bursts}

Two distinct classes of gamma ray bursts have been identified and are known to constitute statistically distinct populations of sources [2]. The long bursts cluster around few tens of seconds of gamma
Properties of short and long GRBs

TABLE I

\begin{tabular}{c|c|c|c}
\hline \hline Type & $\begin{array}{c}\text { Mean } \\
\text { duration }[\mathrm{s}]\end{array}$ & $\begin{array}{c}\text { Peak in energy } \\
\text { spectrum }[\mathrm{keV}]\end{array}$ & Origin \\
\hline long & 25 & $\log \left(E_{\text {peak }}\right)=2.2$ & $\begin{array}{c}\text { collapse of } \\
\text { massive star }\end{array}$ \\
\hline short & 0.7 & $\log \left(E_{\text {peak }}\right)=2.7$ & $\begin{array}{c}\text { merger of } \\
\text { two compact } \\
\text { stars }\end{array}$
\end{tabular}

ray emission, while the short bursts are typically lasting a fraction of a second only. Also, their characteristic spectral energy distributions have distinct characteristics (see Table I).

\subsection{Long gamma ray bursts}

Since the 1990s we have known that the GRBs originate mostly in the distant galaxies and many of them are associated with supernovae. They have to be in fact special supernova types (only 10 per cent of them meet the criteria), because the core of a collapsing star needs to form a black hole, surrounded by a disk composed of the remnant matter from the stellar envelope. It is the accretion of matter onto a rotating black hole that is able to provide the large enough energy to account for the observed properties of GRB phenomena. This process is relatively long (several tens-hundreds seconds).

\subsection{Short gamma ray bursts}

Another mechanism of producing a shorter GRB is the coalescence of two neutron stars. A transient structure is then formed and collapses to a black 
hole. The surrounding remnants of dense matter form a disk composed of elementary particles and neutrinos. The process of disk accretion, mediated by magnetic fields provides power to extract the rotational energy of the black hole and launch a relativistic jet [3]. This collimated outflow is where the gamma rays are produced.

\section{Central engine}

\subsection{Progenitors}

The collapsar scenario is able to explain the longduration GRBs, while the short GRBs are associated with the mergers of compact objects. In the long GRB case, the energetics of the explosion is consistent with the gravitational mass of the progenitor star: $E=G M_{\star}^{2} / R \approx 10^{54} \mathrm{erg}$. The jet has to have a highly relativistic speed, with a bulk Lorentz factor of $\Gamma \approx 100$, in order to be able to break through the stellar envelope. Also, the duration, time variability, and the following afterglow emission of these GRBs at lower energies are consistent with the collapsing massive stars that reside in star-forming host galaxies.

In the short GRB case, the compact binary mergers are almost equally energetic. In the case of the duration of the burst, they are set by the viscous timescale of an accretion disk formed from the tidally-disrupted remnant. This viscous time is given as

$$
t_{\mathrm{vis}} \approx \alpha_{\mathrm{vis}}^{-1} \sqrt{\frac{R_{\mathrm{disk}}^{3}}{G M_{\mathrm{NS}}}}\left(\frac{H}{R_{\mathrm{disk}}}\right)^{-2}
$$

and is about $0.5 \mathrm{~s}$ for typical parameters of such a system.

The highly relativistic speeds of the jets are also supported by the observation of non-thermal energy spectra, which otherwise would not be possible because of a large optical depth due to the electronpositron pair production, e.g. see [4].

The discovery of the binary neutron star (NS-NS) mergers in the gravitational wave observation recently made at LIGO (GW170817 and GW190425), as well as the detection of associated electromagnetic counterparts provided a direct proof of the NS-NS system being the source of short GRBs (see review by [5]). The schematic view of a central engine of a GRB, in a unified approach, is shown in Fig. 1.

\subsection{Numerical modeling of engine and jet structure}

We compute the structure and evolution of black hole accretion disks using the numerical simulations, governed by the equations of general relativistic magnetohydrodynamics. In particular, such disks and outflows can be found at the base of relativistic jets in the extragalactic sources, like blazars or gamma ray bursts. Long-lasting, detailed computations are essential to properly determine the

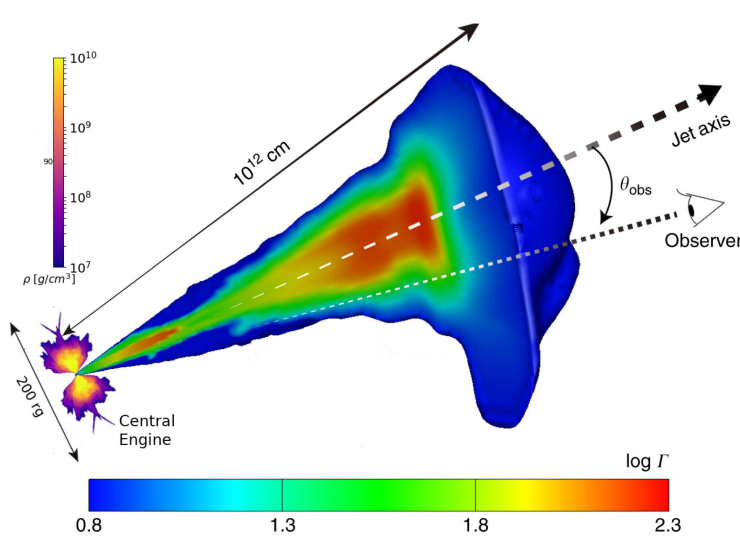

Fig. 1. Schematic idea of GRB. The relativistic jet is ejected (Lorentz factor about 100). Its origin is the "central engine" where a black hole sits. The jet emits gamma ray radiation, collimated towards the observer.

physics of these jets and confront the theoretical models with the observables available from astrophysical observatories in space and from groundbased detectors.

Our numerical scheme works in a conservative manner, by solving a set of non-linear equations at each time-step, to advance the conserved quantities from one time step to the next. The efficiency of computations is enhanced thanks to the code parallelisation. We use the message passing interface (MPI) techniques to distribute the computational grid over the threads. Such calculations are computationally demanding and also required special fine-tuning of the code algorithm.

Our calculations are started with a steady state model of the flow, based on the analytical, equilibrium solution driven by the main physical parameters of the black hole accretion disk (namely, $\mathrm{BH}$ mass, its spin and size and density of the accretion torus). Properties of the pressure equilibrium torus around a black hole, supported by a constant specific angular momentum over radius, were found by [6] and [7].

In the latter model, the angular momentum distribution is chosen to have a power law distribution, which differs from the default disk model of [6], where the angular momentum is assumed to be constant in the disk. This model will allow us to create an initial torus with a large amount of poloidal magnetic flux.

Starting from the Chakrabarti solution, implemented as the initial condition for our simulations, we use a dynamical scheme and we follow the flow evolution. This is achieved by solving numerically the continuity and momentum-energy conservation equations in the GRMHD framework

$$
\begin{aligned}
& \left(\rho u_{\mu}\right)_{; \nu}=0, \\
& T^{\mu \nu}=T_{\text {gas }}^{\mu \nu}+T_{\mathrm{EM}}^{\mu \nu}=0,
\end{aligned}
$$




$$
\begin{aligned}
& T_{\mathrm{gas}}^{\mu \nu}=\rho h u^{\mu} u^{\nu}+p g^{\mu \nu}= \\
& \quad(\rho+u+p) u^{\mu} u^{\nu}+p g^{\mu \nu}, \\
& T_{\mathrm{EM}}^{\mu \nu}=b^{2} u^{\mu} u^{\nu}+\frac{1}{2} b^{2} g^{\mu \nu}-b^{\mu} b^{\nu}, \\
& b^{\mu}=u_{\nu}^{*} F^{\mu \nu} .
\end{aligned}
$$

In fact, we account for both the gas and electromagnetic components of the stress tensor and $u^{\mu}$ is the four-velocity of gas, $u$ is the internal energy, $\rho$ is the density, $p$ denotes the pressure, and $b^{\mu}$ is the magnetic four-vector. Here, $F$ is the Faraday tensor and in the force-free approximation the Lorentz force vanishes and one has $E_{\nu}=u^{\nu} F^{\mu \nu}=0$.

The model system of equations is supplemented with an equation of state, in the polytropic form

$$
p_{g}=K \rho^{\gamma},
$$

where $p_{g}$ is the gas pressure, $\rho$ is the density, $K$ is the constant specific entropy, and the polytropic index $\gamma=4 / 3$ is typically used in the context of gamma ray bursts. The plasma $\beta$ parameter is defined as the ratio of the fluid's thermal to the magnetic pressure, $\beta \equiv p_{g} / p_{\text {mag }}$. For our purpose, we normalize the magnetic field in the torus to have

$$
\beta=\frac{(\gamma-1) u_{\max }}{\left(b_{\max }^{2} / 2\right)},
$$

where $u_{\max }$ is the internal energy at the torus pressure maximum radius $r_{\max }$.

The resulting evolved structure of the GRB central engine is shown in Fig. 2 (disk in Fig. 2a and jet in Fig. 2b). The snapshot is taken at time $t=2000 t_{g}$, where $t_{g}=G M / c^{3}$ denotes geometric time unit and scales with black hole mass. For GRB engine, typical mass of the central black hole is in the range from $M \approx 3$ up to $M \approx 30$ solar masses. Note that in the evolved state, we are able to obtain the physically motivated structure of the accretion torus in the equatorial plane of the rotating black hole and low-density polar funnels, with the dominant magnetic field. These funnels carry both the thermal and Poynting energy along the jet axis. The ultimate bulk Lorentz factor in the jet, achieved at "infinity" (outside the computational domain), will possibly reach the order of magnitude of this total energetics parameter, so $\Gamma \approx$ few hundreds.

\section{Discussion}

Our numerical simulations, performed by the computational astrophysics group at the Center for Theoretical Physics, PAS, provide working tools to model the central engine of both long and short GRBs.

The unified approach is possible because we scale the size and timescale of the system by converting the geometric units to physical ones, as adequate in the GRMHD modeling.

We also notice that the variability timescales, modeled in our simulations as the time variability
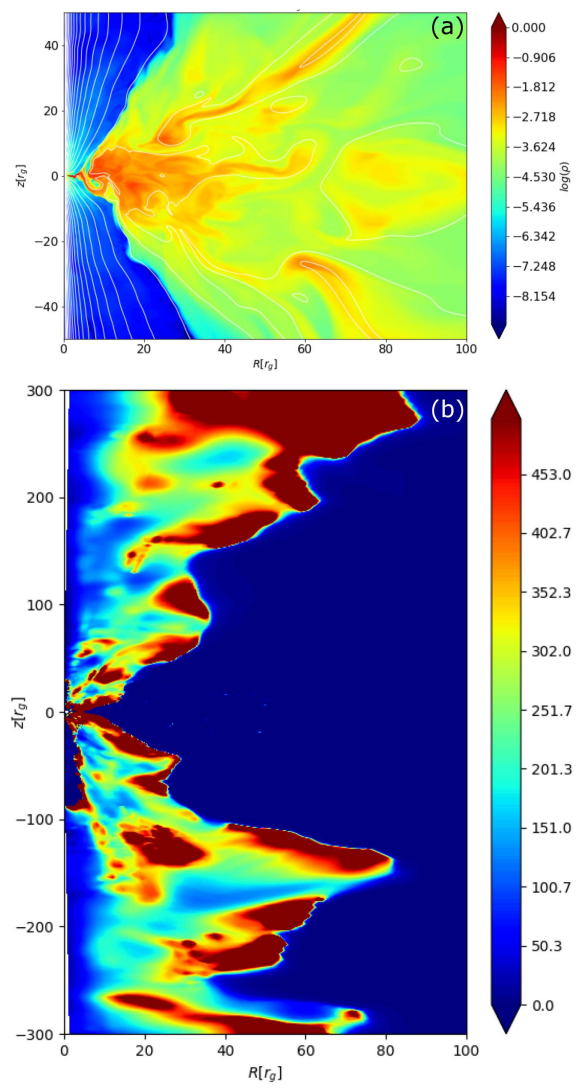

Fig. 2. (a) Distribution of mass density and configuration of magnetic field lines in the region close to the black hole, at the base of a gamma ray burst. (b) Structure of the jet launched from the rotating black hole, in terms of its total energetics (the thermal plus the Poynting energy). The results from an axisymmetric numerical simulation are shown, for black hole spin $a=J / M^{2}=0.7$. The snapshot is taken at time $t=2000 t_{g}$.

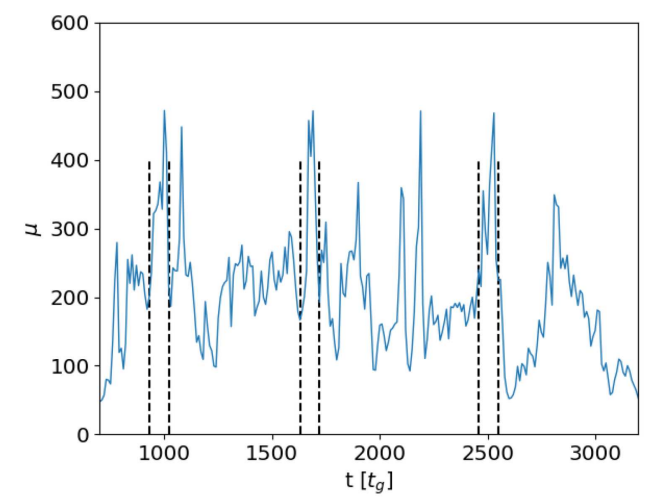

Fig. 3. Time variability of the jet in terms of its total energetics (the thermal plus the Poynting energy), as measured during the simulation at a chosen point in the jet (located at an angle $\theta=5^{\circ}$ from the jet axis). The result of numerical 2dimensional simulation is shown, for black hole spin $a=J / M^{2}=0.7$ and magnetic field normalized in torus with $\beta\left(r_{\max }\right)=60$. The dashed lines show the characteristic timescale of the MRI instability, measured as the maximum growth rate timescale (Gammie 2004) which correlates with the pulse width. 


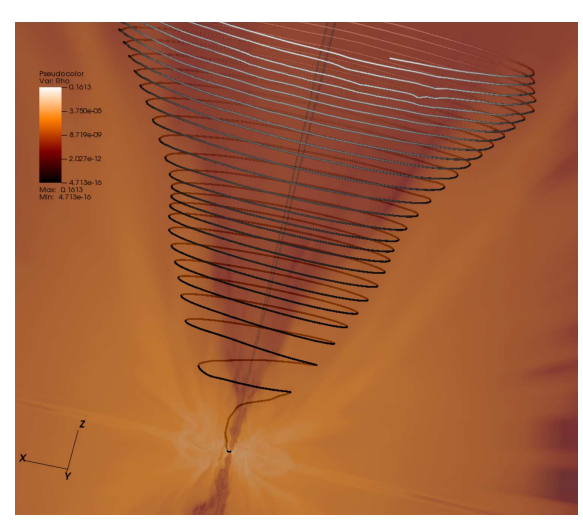

Fig. 4. Structure of the accretion flow and large scale magnetic field in the jet - the result of 3-dimensional numerical simulation. An exemplary field line is depicted as launched from the black hole horizon, with a dominant toroidal field component.

of the jet energetics (see Fig. 3), are governed by the timescale of the magneto-rotational instability in the accretion disk.

In order to properly govern the regime of longduration GRB, with a sustained magnetic turbulence in the engine, full 3-dimensional simulations are needed. This is a work in progress carried out by our group (see Fig. 4).

\section{Conclusions}

The models considered in this paper, i.e., models with a good spatial resolution to solve a physical process close to a black hole, need large computational resources. We already ran several series of calculations on the OKEANOS supercomputer of the Warsaw ICM Supercomputing Center and on the Prometheus supercomputer within the PL-Grid and the work is continued. The purpose is to study the polarimetric signatures of strong magnetic fields near event horizon of a black hole in the Galaxy center [8].

\section{Acknowledgments}

The authors acknowledge financial support from the Polish National Science Center (grants No. 2016/23/B/ST9/03114 and No. 2019/35/B/ST9/04000). We have used the computational resources of the Warsaw ICM through grant Gb79-9 and the PL-Grid through grant grb3.

\section{References}

[1] R.D. Blandford, R.L. Znajek, Mon. Notices Royal Astron. Soc 179, 433 (1977).

[2] C. Kouveliotou, C.A. Meegan, G.J. Fishman, N.P. Bhat, M.S. Briggs, T.M. Koshut, W.S. Paciesas, G.N. Pendleton, Astrophys. J. Lett. 413, L101 (1993).

[3] A. Janiuk, Y.F. Yuan, Astron. Astrophys. 509, A55 (2010).

[4] C. Thompson, Mon. Notices Royal Astron. Soc. 270, 480 (1994).

[5] A. Janiuk, K. Sapountzis, in: Cosmic Rays, Ed. Z. Szadkowski, IntechOpen, Rijeka 2018.

[6] L.G. Fishbone, V. Moncrief, Astrophys. J. 207, 962 (1976).

[7] S.K. Chakrabarti, Astrophys. J. 288, 1 (1985).

[8] M. Mościbrodzka, A. Janiuk, M. De Laurentis arXiv:2103.00267, 2020. 